\title{
A Study of the Electronic Properties of Liquid Alkali Metals. A Self-Consistent Approach
}

\author{
W. Geertsma ${ }^{1}$, D. Gonzalez ${ }^{2}$, and L. H. Gonzalez ${ }^{3}$ \\ ${ }^{1}$ Departamento de Física, Universidade Federal do Espírito Santo \\ Av. Fernando Ferrari s/n, 29060-900, Vitória, ES (Brazil) \\ ${ }^{2,3}$ Departamento de Fisica Teorica, Universidad de Valladolid, \\ E-47011 Valladolid (Spain)
}

Received on 7 August, 2001

\begin{abstract}
We study the electronic properties (density of states, conductivity and thermopower) of some nearly-freeelectron systems: the liquid alkali metals and two liquid alloys, $\mathrm{Li}-\mathrm{Na}$ and $\mathrm{Na}-\mathrm{K}$. The study has been performed within the self-consistent second order Renormalized Propagator Perturbation Expansion (RPE) for the self-energy. The input ionic pseudopotentials and static correlation functions are derived from the neutral pseudoatom method and the modified hypernetted chain theory of liquids, respectively. Reasonable agreement with experiment is found for $\mathrm{Na}, \mathrm{K}, \mathrm{Rb}$ and $\mathrm{Na}-\mathrm{K}$, whereas for $\mathrm{Li}$ and $\mathrm{Cs}$ and $\mathrm{Li}-\mathrm{Na}$ the agreement is less satisfactory.
\end{abstract}

\section{Introduction}

The introduction of the diffraction model [1] together with the development of the pseudopotential concept, marked the beginning of extensive calculations of structural and electronic properties of liquid metals [2,3]. Based on this concept one can justify the use of simple second-order perturbation theory for weak scattering systems like the alkalis. The most used expression in this Nearly-Free Electron (NFE) model is the well-known Ziman formula for the resistivity. Two key ingredients appear in it, namely the static structure factor $S(q)$ and the screened pseudopotential $w(q)$. These two quantities are not independent, since the pseudopotential determines the forces between the ions, which ultimately determine the structure of the liquid. Therefore $S(q)$ should be obtained from $w(q)$ and the result used in the evaluation of the electronic properties, and from the new electronic density one should again derive a new pseudopotential. This is what we call a self-consistent calculation of the electronic properties of a liquid metal.

Most of the calculations up to date are, with the exception of ab-initio Molecular Dynamics simulations, not self-consistent, i.e. for $S(q)$ one takes either one from an analysis of the experimental structure factor data, or one obtained from a model for the interatomic interactions, like hard spheres, with parameters fitted to experimental data. This model is then used as input either in an approximate scheme to solve the Ornstein-Zernike equations like the Percus-Yevick or (Modified) HyperNetted Chain, or in a liquid structure computer simulation. Then a pseudopotential is chosen and the electronic properties, for example the electronic density of states (EDOS), are calculated. There have been some attempts to study the properties in a self-consistent way in a more restricted sense, that is, using the same pseudopotential for the structure factor, thermodynamic properties and conductivity. However up to the present day such a scheme has failed: it leads to disagreement with either the electronic properties, or with the atomic properties.

There are at least two important exceptions in the previous works, which do make the calculations within the spirit of self-consistency. One is the work of Jank and Hafner [5], where molecular dynamics are performed with effective pair potentials derived from a given pseudopotential, and then some ionic configuration is selected to perform a Linearized-Muffin-Tin-Orbitals electronic calculation.

The other calculations, which are self-consistent, are the modern $a b$-initio molecular dynamics (AIMD) simulations. Using this method, we are only aware, in the case of alkali metals, of EDOS and conductivity calculations for $\mathrm{Na}$ and $\mathrm{Rb}$ at several temperatures $[7,8]$.

In this work, we propose a completely different method, which does not rely on simulations, to obtain within a self-consistent scheme, the ionic structure and electronic properties of the liquid metal. Moreover, the theory for the calculations of the electronic properties which is the second-order Renormalized Propagator Perturbation Expansion (RPE) [3,4], satisfies the so-called Generalized Optical Theorem (GOT), which provides a second level of selfconsistency in the theory, namely between the EDOS on the one hand and the conductivity on the other hand. This method is much faster and much less demanding than AIMD simulations, but, as we will show below, achieves the same level of agreement with experiment for weak scattering systems, like the alkali metals, when pseudopotentials of similar quality are used. 
A similar RPE-type scheme to calculate the electronic structure has been applied before but only in a non selfconsistent way (see for a discussion $[2,3,4]$ ): the integral equation for the self-energy was approximated by neglecting the self-energy in the right hand side of eq. 2.3 below.

The bare pseudopotentials used in this work were constructed from first principles using the Neutral Pseudo Atom (NPA) method: one solves a system of one alkali atom immersed in an electron jellium in the Local Density Approximation (LDA). Linear Response Theory (LRT) was then applied to obtain the screened pseudopotential $w(q)$ on the one hand and the effective pair potential on the other hand; for details see $[13,14]$. The structure factor $S(q)$ was obtained from the pair potential using the Modified HyperNetted Chain (MHNC) theory of liquids. This combination has already been applied successfully to the study of the static and dynamic structure factors of the liquid alkalis as well as the Na-K and Li-Na liquid alloys [13, 14], the systems studied in this paper.

\section{Theory}

We give here the essentials of the theory, further details can be found in [4]. Atomic units with $2 m=1, \hbar=1, e^{2}=1$ are used throughout.

We consider a system of $N$ ions with their corresponding valence electrons in a volume $\Omega$. Each valence electron moves in a self-consistent potential due to the ion cores and the other valence electrons. Therefore, we can write the single-particle Hamiltonian as: $\mathcal{H}=\mathcal{H}_{0}-\nu$, where $\mathcal{H}_{0}$ is the kinetic energy operator and $\nu(\mathbf{r})=\Sigma_{i} w\left(\left|\mathbf{r}-\mathbf{R}_{i}\right|\right)$ is effective one-electron potential which is taken to be the sum of spherically symmetric screened local pseudopotentials centered on each ion. The central quantity in this study is the one-electron Green function, $G(\mathbf{k}, E)$. The EDOS per atom, imit energy and unit volume is given by

$$
n(E)=-\frac{1}{\pi} \int \frac{d}{(2 \pi)^{3}} \operatorname{Im} G(\mathbf{k}, E)
$$

Moreover in terms of the so-called self-energy, $\Sigma(\mathbf{k}, E)$, the Green function can also be expressed as

$$
G(\mathbf{k}, E)=\left(E-k^{2}-\Sigma(\mathbf{k}, E)\right)^{-1}
$$

Within the second-order RPE [4], the self-energy is approximated by

$$
\Sigma(\mathbf{k}, E)=\rho \int \frac{d \mathbf{k}^{\prime}}{(2 \pi)^{3}} w^{2}\left(\left|\mathbf{k}-\mathbf{k}^{\prime}\right|\right) S\left(\left|\mathbf{k}-\mathbf{k}^{\prime}\right|\right) G\left(\mathbf{k}^{\prime}, E\right),
$$

where a constant term $\rho w(q=0)$ has been dropped using it as the energy origin, and $\rho$ denotes the atomic number density. This expression for the self-energy is easily extended to liquid alloys by the substitution of $w^{2}(q) S(q)$ by $\Sigma_{i, j}\left(x_{i} x_{j}\right)^{1 / 2} w_{i} t(q) w_{j}(q) S_{i j}(q)$, where $x_{i}$ and stands for the concentration and $w_{i}(q)$ for the screened pseudopotential of the $i$ th-component, whereas $S_{i j}(q)$ are the AshcroftLangreth partial static structure factors.

Substituting the relation (2.2) into (2.3), an integral equation is obtained for the self-energy, which is solved for each energy, obtaining $\Sigma(\mathbf{k}, E), G(\mathbf{k}, E)$, and $n(E)$ for that energy from equation (2.1).

The static electrical conductivity is given by the KuboGreenwood equation

$$
\sigma=\int d E\left(-\partial f_{F D}(E) / \partial E\right) \sigma(E)
$$

where $f_{F D}(E)$ is the Fermi-Dirac distribution function and $\sigma(E)$ is the contribution of the electrons of energy $E$ to the conductivity, which is given by

$$
\sigma(E)=\frac{8}{3 \pi} \int \frac{d \mathbf{k}}{(2 \pi)^{3}}\left(K(\mathbf{k}, E)-\frac{1}{2} \mathbf{k} \Delta_{\mathbf{k}} \operatorname{Re} G(\mathbf{k}, E)\right),
$$

where $K(\mathbf{k}, E)$ obeys the following integral equation [6]

$$
\frac{K(\mathbf{k}, E)}{|G(\mathbf{k}, E)|^{2}}=k^{2}+\int \frac{d \mathbf{k}^{\prime}}{(2 \pi)^{3}} \Lambda\left(\mathbf{k}, \mathbf{k}^{\prime}, E\right) \frac{\mathbf{k} \dot{\mathbf{k}}^{\prime}}{k^{\prime 2}} K\left(\mathbf{k}^{\prime}, E\right)
$$

The vertex function, $\Lambda\left(\mathbf{k}, \mathbf{k}^{\prime}, E\right)$, has still to be specified. A self-consistent calculation of the EDOS and the conductivity requires the self-energy and the vertex function to be related by the generalized optical theorem (GOT)

$$
\operatorname{Im} \Sigma(\mathbf{k}, E)=\int \frac{d \mathbf{k}^{\prime}}{(2 \pi)^{3}} \Lambda\left(\mathbf{k}, \mathbf{k}^{\prime}, E\right) \operatorname{Im} G\left(\mathbf{k}^{\prime}, E\right)
$$

We have chosen

$$
\Lambda\left(\mathbf{k}, \mathbf{k}^{\prime}, E\right)=\rho w^{2}\left(\left|\mathbf{k}-\mathbf{k}^{\prime}\right|\right) S\left(\left|\mathbf{k}-\mathbf{k}^{\prime}\right|\right)
$$

which clearly satisfies the GOT when the second-order RPE expansion is made for the self-energy 2.3.

Finally, once $\sigma(E)$ has been obtained, the thermopower, $S$, can be calculated as a function of energy from the Mott relation

$$
S=-\frac{\pi^{2} k_{B}^{2} T}{3|e|} \frac{d \log \sigma(E)}{d E}
$$

\section{Numerical details}

For the calculation of the self-energy we used a linear mesh of about 400 points with a cut-off of the pseudopotential at $4 k_{F}$. Results of the various electronic properties are well converged because a twice as high cut-off does give results which differ only by about .1\%. That such a cut-off is sufficient also follows from the observation that the second contribution to the conductivity is nearly independent of the energy. 
The rather singular integrand appearing in the expressions for the self-energy and the conductivity was calculated using a rational expansion up to second order in $q$ in each interval of the $q$-mesh, and were evaluated analytically. Checks using various integration schemes showed that this scheme gives a very reliable approximation of these $q$ integrals, with an estimated error of $.1 \%$ or less.

The iteration was started from the bottom of the band. Convergence of the self-energy was obtained within 10 iterations - irrespective of its initialization. No mixing scheme was used. The convergence of the integral equation for the conductivity was rather slow near the bottom of the band, requiring some 20 to 50 iterations. For the DOS as a function of the energy from the bottom of the band to about $2 E_{F}$ we used about 50 to 100 energy points. The solution of the integral equation for the conductivity showed oscillations as a function of energy when the $q$-mesh is taken too coarse.

\section{Results and discussion}

The preceding formalism has been applied to liquid alkali metals at thermodynamic conditions near the melting point as well as to liquid $\mathrm{Li}-\mathrm{Na}$ and $\mathrm{Na}-\mathrm{K}$ alloys at the temperatures $T=725 \mathrm{~K}$ and $T=373 \mathrm{~K}$ respectively. Calculations were also performed for the thermodynamic states considered by Silvestrelli et al. [7] for liquid $\mathrm{Na}$ and Shimojo et al. for liquid $\mathrm{Rb}$ [8].

The results obtained for the EDOS of the liquid alkalis, and liquid Li-Na and Na-K alloys are shown in Fig. 1. First, we note that the present results are, in general, very similar to those derived from the free-electron model and only for $\mathrm{Rb}$ and $\mathrm{Cs}$ we see some structure appearing in the EDOS. The bottom of the band is only slightly shifted below the free-electron band; this shift increases on alloying from about $0.003 \mathrm{Ry}$. for the pure elements to $0.02 \mathrm{Ry}$ for the alloys. We checked the validity of these results of the RPE by comparing it with results of the Quasi-Crystalline approximation (QCA) [3] and found only small changes in the total EDOS with respect to the RPE results.

The valence bands of the alkalis have been investigated by Indlekofer and Oelhafen [9] using various UPS excitation energies. There are two important characteristics observed in these valence band spectra: (i) the widths of the valence bands, i.e. the energy difference between the Fermi level and the bottom of the valence band, are narrower than the widths expected from free-electron behaviour, by some 15 to $25 \%$, and (ii) the spectra show a triangular shape, except for Li where it is parabolic. The same narrowing is observed in the crystalline state of alkalis [10], and has been attributed to electron correlation effects not taken into account in the Local Density Approximation [11].

The triangular shape has been shown to be consistent with a free-electron EDOS, and is explained by the difference in cross sections of the valence electrons with different angular momentum for excitations in the UPS regime [5]. In the RPE these different angular moment components are not available.

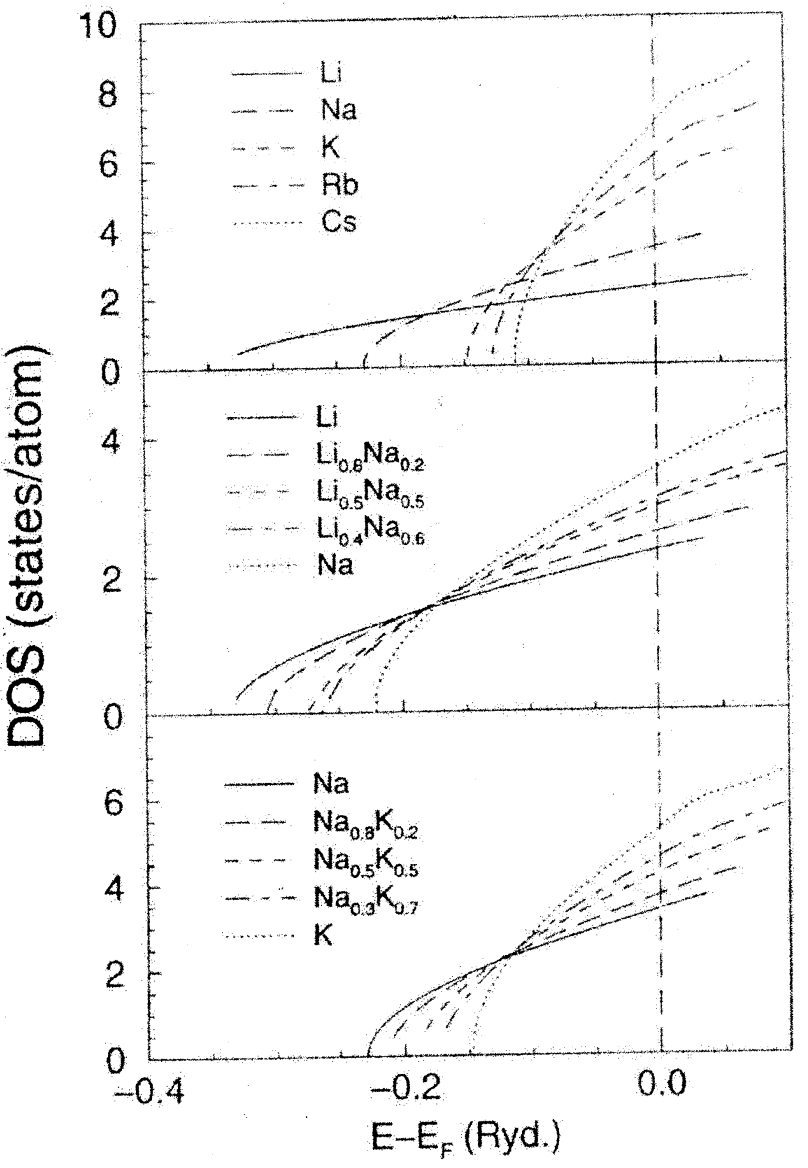

Figure 1. The EDOS as a function of energy for the liquid alkalis near the melting point, and for the liquid alloys $\mathrm{Li}-\mathrm{Na}$ at $\mathrm{T}=725 \mathrm{~K}$ and $\mathrm{Na}-\mathrm{K}$ at $\mathrm{T}=373 \mathrm{~K}$.

Although the present scheme is not able to account for the bandwidth reduction of the EDOS, it must be remarked that this is also the case with the AIMD simulations reported for liquid $\mathrm{Na}$ [7] and $\mathrm{Rb}$ [8]. In both cases, the valence bands show an almost parabolic shape and a width similar to the free-electron model.

The electrical conductivity is calculated by solving the integral equation 2.6 for $K(\mathbf{k}, F)$ and using the vertex defined in 2.8. The electrical conductivity as a function of energy behaves in a similar way for all the systems studied. It is small near the bottom of the band, and then it increases to a broad maximiun around the Fermi level, and then it decreases. The Fermi level is in all cases located before the maximum in $\sigma(F)$, so according to relation 2.9 leading always to a negative thermopower (see table 1). The sign of the thermopower agrees with the experimental values except for $\mathrm{Li}$ and $\mathrm{Cs}$.

In table 1 we present the resistivity we calculated in the present RPE scheme together with those predicted by $\mathrm{Zi}$ man's formula using the same structure factor and pseudopotential. The values of the latter approach are in most cases somewhat smaller than the RPE. The comparison with experimental data reveals discrepancies for $\mathrm{Li}$ and $\mathrm{Cs}$, however, the discrepancies are smaller in the case of alloys. 
Table 1: Calculated resistivity $(\mu \Omega \mathrm{cm})$ and thermopower $(\mu \mathrm{V} / \mathrm{K})$ for the liquid alkali metals near melting, the liquid alloys $\mathrm{Li}-\mathrm{Na}$ at $\mathrm{T}=725 \mathrm{~K}$ and $\mathrm{Na}-\mathrm{K}$ at $\mathrm{T}=373 \mathrm{~K}$.

\begin{tabular}{|c|c|c|c|c|c|}
\hline \hline & $\rho$ (Kubo) & $\rho$ (Ziman) & $\rho$ (exp) & S(Kubo) & S(exp) \\
\hline $\mathrm{Li}$ & 7.3 & 7.0 & 24 & -3.9 & +21.7 \\
$\mathrm{Na}$ & 16.3 & 15.8 & 9.6 & -8.0 & -7.9 \\
$\mathrm{~K}$ & 19.6 & 18.5 & 13.0 & -10.6 & -14.0 \\
$\mathrm{Rb}$ & 22.0 & 20.8 & 22.0 & -11.2 & -6.3 \\
$\mathrm{Cs}$ & 14.9 & 13.8 & 36.0 & -11.7 & +6.4 \\
\hline $\mathrm{Na}$ & 36.2 & 36.4 & 24.6 & -16.0 & -13.2 \\
$\mathrm{Li}_{0,4} \mathrm{Na}_{0.6}$ & 27.8 & 27.5 & 30.2 & -13.9 & +2.2 \\
$\mathrm{Li}_{0.5} \mathrm{Na}_{0.5}$ & 25.9 & 25.3 & 31.5 & -13.4 & +5.1 \\
$\mathrm{Li}_{0.6} \mathrm{Na}_{0.4}$ & 23.2 & 22.8 & 32.2 & -12.6 & +8.5 \\
$\mathrm{Li}_{0.8} \mathrm{Na}_{0.2}$ & 18.2 & 17.8 & 33.1 & -11.1 & +16.2 \\
$\mathrm{Li}$ & 12.3 & 12.0 & 33.6 & -9.0 & +25.2 \\
\hline $\mathrm{K}$ & 21.6 & 20.3 & 14.9 & -11.5 & - \\
$\mathrm{Na}_{0.3} \mathrm{~K}_{0.7}$ & 48.4 & 48.2 & 39.5 & -8.5 & - \\
$\mathrm{Na}_{0.5} \mathrm{~K}_{0.5}$ & 48.4 & 49.0 & 43.0 & -8.6 & - \\
$\mathrm{Na}_{0.8} \mathrm{~K}_{0.2}$ & 32.6 & 32.8 & 26.0 & -8.3 & - \\
$\mathrm{Na}$ & 16.3 & 15.8 & 9.6 & -8.0 & - \\
\hline \hline
\end{tabular}

In the case of $\mathrm{Na}$ at different temperatures we find that, although the structure factor is well reproduced, the values obtained for the conductivity are always smaller than the experimental ones, the discrepancy with experiment becoming smaller as the temperature increases. The same behaviour for the resistivity is obtained by Silvestrelli et al. [7] in their AIMD studies for Na; moreover, the discrepancy with experiment is in their AIMD calculation larger for lower temperatures than in the RPE. They used the Topp-Hopfield pseudopotential for $\mathrm{Na}$, which reproduced correctly the structure factor in all thermodynamic states. Moreover they performed computations with norm-conserving non-local pseudopotentials, even including core corrections, but no improvement was obtained for the conductivity values, compared with experiment.

For Rb, the AIMD of Shimojo et al. [8], using a normconserving non-local Troullier-Martins pseudopotential, obtained conductivities which are much smaller than the experimental ones, whereas our calculations agree very well with experiment. It is also interesting to note that the structure factor was correctly reproduced both in the AIMD and in our calculations.

We also performed calculations for the atomic and electronic properties using the Fiolhais-Perdew pseudopotential [15], but did not obtain improved agreement with experiment.

\section{Conclusions}

The above comparisons show that when suitable pseudopotentials are used, the combination of LRT, MHNC and RPE produces self-consistent results for the atomic and electronic properties of liquid alkali metals, which agree with modern state of the art AIMD methods, at least for alkalis for which results using both these methods are available. The agreement of our atomic structure factors with experiment is excellent. The agreement between theoretical and experimental electronic properties is less satisfactory. For Li and Cs, we find large discrepancies with experimental data for the resistivity and for thermopower of $\mathrm{Li}$ and $\mathrm{Cs}$ for we do not even find the right sign. No thermopower data are available from these AIMD simulations.

In our calculations we have found that for the pure alkali metals, the deviation of the EDOS from the free-electron parabola is rather small, and it increases somewhat on alloying. The resistivity and thermopower agree reasonably well with experiment for $\mathrm{Na}, \mathrm{K}, \mathrm{Rb}$ and $\mathrm{Na}-\mathrm{K}$ but is less satisfactory for $\mathrm{Li}$ and $\mathrm{Cs}$, and as a consequence for the liquid $\mathrm{Li}-\mathrm{Na}$ alloys in the Li-rich composition regime. It is known that the pseudopotential of $\mathrm{Li}$ and $\mathrm{Cs}$ has rather large non-local components, for Li because $p$ states are missing in the core, and for Cs because d states are present near the Fermi level. Such nonlocal components of the pseudopotential do not seem to contribute much to the atomic structure, but could be of importance for the electronic properties. However, the use of a non-local pseudopotential by Shimojo [8] does not improve the AIMD result for the conductivity for $\mathrm{Rb}$ when compared with experiment. Note that for $\mathrm{Rb}$ we find good agreement with experiment. Such non-local components could alter the position of the maximum in $\sigma$ as a function of energy. Introducing relative stronger electron-ion scattering below the Fermi level, which would give a minimum in $\sigma(E)$, thus also changing the sign of the thermopower.

The larger bandwidth calculated for the EDOS compared with experiment has been attributed to electron correlations not taken into account in the LDA approximation. In our scheme we take these electron-electron interactions into account implicitly in the screened pseudopotential. To take 
these electron-electron interactions into account in a more explicit way would require the introduction of a self-energy in the r.h.s. of equation 2.3, and using the bare electron-ion potential: in terms of diagrams it means that instead of summing part of the electron-electron interactions in a screened electron-ion potential, one collects them in a medium propagator, which describes the motion of electrons including the electron-electron interactions between two scattering events on bare electron-ion potentials. This electron self-energy can be approximated for these NFE systems in the Random Phase Approximation (see [16] . The consequence of such a scheme for the conductivity is not an increase of the effective electron-ion scattering, but could result in a slight change of the EDOS at the Fermi level. Most of the changes in the EDOS should occur below the Fermi level. The EDOS at the Fermi level of $\mathrm{Na}$ is nearly given by the NFE model.

The discrepancy of the electronic transport properties with experimental results we attribute to the construction of the pseudopotential, and not to basic problems in our theory which should be valid for these weak scattering systems, as also follows from the comparison of our results for the resistivity and those obtained from the Ziman equation: we find only small corrections to the Ziman results.

The success of both the present approach and the AIMD in reproducing the experimental electronic properties appears to depend very much on the subtle details of the pseudopotential. The atomic structure and electronic structure and transport properties are determined by different parts of the pseudopotential. Electrons penetrate more into the core region and are also more sensitive to angular non-local components. So changes in these contributions not necessarily will affect the atomic structure.

The conclusion is that there exists accurate theoretical tools that can in principle give reliable self-consistent results for both the atomic and electronic properties of liquid (alkali) metals provided adequate pseudopotentials are used. However, considering the results of the present calculations, we conclude that most of the pseudopotentials proposed up to now for alkali metals, although good enough to describe the static and dynamic atomic structure, are not accurate enough to give good agreement also with the experimental electronic properties. Even for $\mathrm{Na}$, which has always been considered as the simplest metal to describe.

In contrast to the pseudopotentials used in AIMD simulations our pseudopotentials are adjusted to the electron density in the liquid metal. We attribute the failure of our pseudopotentials to describe the EDOS to the linear screening approximation. Further checks are currently being undertaken. Preliminary results for a pseudopotential based on the electronic density show better agreement with experiment for the electronic properties, while preserving the good agreement of the atomic properties with experiment. We attribute the discrepancy between experiment and theory in case of the electronic transport properties to so-called nonlocal contributions to the pseudopotential, which in case of $\mathrm{Li}$ and $\mathrm{Cs}$ can be rather large.

We have shown that fully self-consistent $a b$-initio calculations, with as only input the chosen pseudopotential, using integral equation approximations for the static and dynamic atomic structure as well as for the electronic properties give results of the same reliability as AIMD simulations, under the condition that the electron-ion pseudopotential has been constructed well. The advantage of the present method is that it is much faster than the AIMD simulations and only requires minimal computational resources, and so the study of possible improvements in the construction of the pseudopotential taking into account nonlocal components and implementation of a scheme bare electron-ion potentials together with the electron-electron self-energy can in principle easily be implemented and studied.

\section{Acknowledgments}

Work supported by the DGES (Grant PB95-0720C0201), Junta de Castilla y Leon (Grant VA63/96) and the European Community TMR contract ERBFMBICT-950218 and from, and a grant from CNPq (30092897-0) during the final stages of this work.

\section{References}

[1] J. M. Ziman, Phil. Mag. 6, 1031 (1961).

[2] M. Shimoji, Liquid Metals, Academic Press (1977).

[3] L. E. Ballentine, Adv. Chem. Phys. 31, 263 (1975).

[4] A. B. van Oosten and W. Geertsma, Physica B (1985) 5563; W. Geertsma and W. van der Lugt, Can J. Phys. 65, 326 (1987).

[5] W. Jank and J. Hafner, J. Phys.: Condens. Matter 2, 5065 (1990); 26947 (1990).

[6] A. Bringer, Thesis, University of Küln (1970); A. Bringer and D. Wagner Z. Phys. 241, 295 (1971).

[7] P. L. Silvestrelli, A. Alavi and M. Parrinello, Phys. Rev. B 55, 15515 (1997).

[8] F. Shimojo, Y. Zempo, K. Hoshino and M. Watabe, J. NonCryst. Solids 205-207, 893 (1996).

[9] G. Indlekofer and P. Oelhafen, J. Non-Cryst. Solids, 117-118, 340 (1990); 156-158, 226 (1993).

[10] E. Jensen and E. W. Plummer Phys. Rev. Lett. 55, 1912 (1985).

[11] J. E. Northrup, M. S. Hybertsen and G. Louie, Phys. Rev. Lett. 59, 819 (1987).

[12] K. W.-K. Shung and G. D. Mahan, Phys. Rev. Lett. 57, 1076 (1986).

[13] L. E. Gonzalez, D. J. Gonzalez and K. Hoshino, J. Phys. Cond. Matter, 5, 9261 (1993); 8, 4465 (1996).

[14] L. E. Gonzalez, D. J. Gonzalez and M. Silbert, J. Non-Cryst. Solids, 205-207, 443 (1996).

[15] C. Rolhais, J. P. Perdew, S. Q. Armster, J. M. MacLaren and M. Brajczewska, Phys. Rev. B 51, 14001 (1995).

[16] L. Hedin, Phys. Rev. A 139, 796(1995).

[17] R. R. Ohse (ed.) Handbook of Tharmodynamic and Transport of Alkali Metals Blackwell (1985); C. van der Marel and W. van der Lugt, Phys. Rev. B 22, 6030 (1980). 